\title{
Spatial-Temporal Dynamics in Nonlocal Epidemiological Models ${ }^{\star}$
}

\author{
SHIGUi RUAN \\ Department of Mathematics, University of Miami, P. O. Box 249085, Coral \\ Gables, FL 33124-4250, USA
}

\section{Introduction}

Throughout recorded history, nonindigenous vectors that arrive, establish, and spread in new areas have fomented epidemics of human diseases such as malaria, yellow fever, typhus, plague, and West Nile (Lounibos 2002). The spatial spread of newly introduced diseases is a subject of continuing interest to both theoreticians and empiricists. One strand of theoretical developments (e.g., Kendall 1965; Aronson and Weinberger 1975; Murray 1989) built on the pioneering work of Fisher (1937) and Kolmogorov et al. (1937) based on a logistic reaction-diffusion model to investigate the spread of an advantageous gene in a spatially extended population. With initial conditions corresponding to a spatially localized introduction, such models predict the eventual establishment of a well-defined invasion front which divides the invaded and uninvaded regions and moves into the uninvaded region with constant velocity.

Provided that very small populations grow in the same way or faster than larger ones, the velocity at which an epidemic front moves is set by the rate of divergence from the (unstable) disease-free state, and can thus be determined by linear methods (e.g., Murray 1989). These techniques have been refined by Diekmann (1978, 1979), Thieme (1977a, 1977b, 1979), van den Bosch et al. (1990), etc. who used a closely related renewal equation formalism to facilitate the inclusion of latent periods and more general and realistic transport models. Behind the epidemic front, most epidemic models settle to a spatially homogeneous equilibrium state in which all populations co-exist at finite abundances. In many cases, the passage from the epidemic front to co-existence passes through conditions where the local abundances of some or all of the players drops to truly microscopic levels. Local rekindling of the disease usually takes place not only because of the immigration of infectives but also by in situ infections produced by the non-biological remnants of previous populations.

\footnotetext{
* Research was partially supported by NSF grant DMS-0412047, NIH Grant P20-RR020770-01, the MITACS of Canada and a Small Grant Award at the University of Miami.
} 
Kermack and McKendrik (1927) proposed a simple deterministic model of a directly transmitted viral or bacterial agent in a closed population consisting of susceptibles, infectives, and recovereds. Their model leads to a nonlinear integral equation which has been studied extensively. The deterministic model of Barlett (1956) predicts a wave of infection moving out from the initial source of infection. Kendall (1957) generalized the Kermack-McKendrik model to a space-dependent integro-differential equation. Aronson (1977) argued that the three-component Kendall model can be reduced to a scalar one and extended the concept of asymptotic speed of propagation developed in Aronson and Weinberger (1975) to the scalar epidemic model. The Kendall model assumes that the infected individuals become immediately infectious and does not take into account the fact that most infectious diseases have an incubation period. Taking the incubation period into consideration, Diekmann $(1978,1979)$ and Thieme (1977a, 1977b, 1979) simultaneously proposed a nonlinear (double) integral equation model and extended Aronson and Weinberger's concept of asymptotic speed of propagation to such models. All these models are integral equations in which the spatial migration of the population or host was not explicitly modelled.

Spatial heterogeneities can be included by adding an immigration term where infective individuals enter the system at a constant rate. De Mottoni et al. (1979) and Busenberg and Travis (1983) considered a population in an open bounded region and assumed that the susceptible, infective, and removed individuals can migrate inside the region according to the rules of group migration. The existence of traveling waves in epidemic models described by reaction-diffusion systems has been extensively studied by many researchers, for example, Thieme (1980), Källen et al. (1985), Murray et al. (1986) and Murray and Seward (1992) studied the spatial spread of rabies in fox; Abramson et al. (2003) considered traveling waves of infection in the Hantavirus epidemics; Cruickshank et al. (1999), Djebali (2001), Hosono and Ilyas (1995) investigated the traveling waves in general SI epidemic models; Caraco et al. (2002) studied the spatial velocity of the epidemic of lyme disease; Greenfell et al. (2001) discussed the traveling waves in measles epidemics; etc.

In this article we try to provide a short survey on the spatial-temporal dynamics of nonlocal epidemiological models, include the classical KermackMcKendrick model, the Kendall model given by differential and integral equations, the Diekmann-Thieme model described by a double integral equation, the diffusive integral equations proposed by De Mottoni et al. (1979) and Busenberg and Travis (1983), a vector-disease model described by a diffusive double integral equation (Ruan and Xiao 2004), etc. 


\section{Kermack-McKendrick Model}

Kermack and McKendrick (1927) proposed a simple deterministic model of a directly transmitted viral or bacterial agent in a closed population based on the following assumptions: (i) a single infection triggers an autonomous process within the host; (ii) the disease results in either complete immunity or death; (iii) contacts are according to the law of mass-action; (iv) all individuals are equally susceptible; (v) the population is closed in the sense that at the time-scale of disease transmission the inflow of new susceptibles into the population is negligible; (vi) the population size is large enough to warrant a deterministic description.

Let $S(t)$ denote the (spatial) density of individuals who are susceptible to a disease, that is, who are not yet infected at time $t$. Let $A(\theta)$ represent the expected infectivity of an individual who became infected $\theta$ time units ago. If $\frac{d S}{d t}(t)$ is the incidence at time $t$, then $\frac{d S}{d t}(t-\theta)$ is the number of individuals arising per time unit at time $t$ who have been infected for $\theta$ time units. The original Kermack-McKendrick model is the following integral differential equation

$$
\frac{d S}{d t}=S(t) \int_{0}^{\infty} A(\theta) \frac{d S}{d t}(t-\theta) d \theta
$$

Kermack and McKendrick (1927) derived an invasion criterion based on the linearization of equation (1). Assume $S(0)=S_{0}$, the density of the population at the beginning of the epidemic with everyone susceptible. Suppose the solution of the linearized equation at $S_{0}$ has the form $c e^{r t}$. Then the characteristic equation is

$$
1=S_{0} \int_{0}^{\infty} A(\theta) e^{-r \theta} d \theta
$$

Define

$$
R_{0}=S_{0} \int_{0}^{\infty} A(\theta) d \theta
$$

Here, $R_{0}$ is the number of secondary cases produced by one typical primary case and describes the growth of the epidemic in the initial phase on a generation phase. Since $A(\theta)$ is positive, we have $r>0$ if and only if $R_{0}>1$. Therefore, the invasion criterion is $R_{0}>1$.

If the kernel $A(\theta)$ takes the special form $\beta e^{-\gamma \theta}$, where $\beta>0, \gamma>0$ are constants, and define

$$
I(t)=-\frac{1}{\beta} \int_{0}^{\infty} A(\theta) \frac{d S}{d t}(t-\theta) d \theta .
$$

Then $I(t)$ represents the number of infected individuals at time $t$. Let $R(t)$ denote the number of individuals who have been infected and then removed from the possibility of being infected again or of spreading infection. Thus, 
$\frac{d R}{d t}=\gamma I(t)$. This, together with (1) and differentiation of $I(t)$, yields the following ODE system

$$
\begin{aligned}
& \frac{d S}{d t}=-\beta S(t) I(t), \\
& \frac{d I}{d t}=\beta S(t) I(t)-\gamma I(t), \\
& \frac{d R}{d t}=\gamma I(t) .
\end{aligned}
$$

Remark 1. Interestingly, it is system (2) (instead of the original equation (1)) that is widely referred as the Kermack-McKendrick model. Though Kermack and McKendrick (1927) studied the special case (2), a more general version was indeed previously considered by Ross and Hudson (1917) (see Diekmann et al. 1995).

Observe from system (2) that $\frac{d S}{d t}<0$ for all $t \geq 0$ and $\frac{d I}{d t}>0$ if and only if $S(t)>\gamma / \beta$. Thus, $I(t)$ increases so long as $S(t)>\gamma / \beta$, but $S(t)$ decreases for all $t \geq 0$, it follows that $I(t)$ eventually decreases and approaches zero. Define the basic reproduction number as

$$
R_{0}=\frac{\beta S(0)}{\gamma}
$$

If $R_{0}>1$, then $I(t)$ first increases to a maximum attained when $S(t)=\gamma / \beta$ and then decreases to zero (epidemic). If $R_{0}<1$, then $I(t)$ decreases to zero (no epidemic).

The Kermack-McKendrick model and the threshold result derived from it have played a pivotal role in subsequent developments in the study of the transmission dynamics of infective diseases (Anderson and May 1991; Brauer and Castillo-Chavez 2000; Diekmann and Heesterbeek 2000; Hethcote 2000; Thieme 2003).

\section{Kendall Model}

Kendall $(1957,1965)$ generalized the Kermack-McKendrick model to a spacedependent integro-differential equation. Denote $\mathbb{R}=(-\infty, \infty), \mathbb{R}_{+}=[0, \infty)$. Let $S(x, t), I(x, t)$ and $R(x, t)$ denote the local densities of the susceptible, infected, and removed individuals at time $t$ in the location $x \in \mathbb{R}$ with $S+I+R$ independent of $t$. All infected individuals are assumed to be infectious and the rate of infection is given by

$$
\beta \int_{-\infty}^{\infty} I(y, t) K(x-y) d y,
$$


where $\beta>0$ is a constant and the kernel $K(x-y)>0$ weights the contributions of the infected individuals at location $y$ to the infection of susceptible individuals at location $x$. It is assumed that

$$
\int_{-\infty}^{\infty} K(y) d y=1
$$

Removed individuals can be regarded as being either immune or dead and the rate of removal is assumed to be $\gamma I(x, t)$, where $\gamma>0$ is a constant. With this notation, Kendall's model is

$$
\begin{aligned}
& \frac{\partial S}{\partial t}=-\beta S(x, t) \int_{-\infty}^{\infty} I(y, t) K(x-y) d y, \\
& \frac{\partial I}{\partial t}=\beta S(x, t) \int_{-\infty}^{\infty} I(y, t) K(x-y) d y-\gamma I(x, t), \\
& \frac{\partial R}{\partial t}=\gamma I(x, t) .
\end{aligned}
$$

Given a spatially inhomogeneous epidemic model it is very natural to look for traveling wave solutions. The basic idea is that a spatially inhomogeneous epidemic model can give rise to a moving zone of transition from an infective state to a disease-free state. A traveling wave solution of system (3) takes the form $(S(x-c t, t), I(x-c t, t), R(x-c t, t))$. Kendall (1965) proved the existence of a positive number $c^{*}$ such that the model admits traveling wave solutions of all speeds $c \geq c^{*}$ and no traveling wave solutions with speeds less than $c^{*}$. Mollison (1972) studied Kendall's original model in the special case in which there are no removals. With this assumption the system of integro-differential equations reduces to a single equation. For a particular choice of the averaging kernel Mollison (1972) proved the analog of Kendall's result. Atkinson and Reuter (1976) analyzed the full Kendall model for a general class of averaging kernels and obtained a criterion for the existence of a critical speed $c^{*}>0$ and the existence of traveling waves of all speeds $c>c^{*}$. See also Barbour (1977), Brown and Carr (1977), Medlock and Kot (2003), etc.

Minimal wave speeds analogous to those found by Kendall and others also occur in the classical work of Fisher (1937) and Kolmogoroff et al. (1937) concerning the advance of advantageous genes. Aronson and Weinberger $(1975,1978)$ showed that the minimal wave speed is the asymptotic speed of propagation of disturbances from the steady state for Fisher's equation. Roughly speaking, $c^{*}>0$ is called the asymptotic speed if for any $c_{1}, c_{2}$ with $0<c_{1}<c^{*}<c_{2}$, the solution tends to zero uniformly in the region $|x| \geq c_{2} t$, whereas it is bounded away from zero uniformly in the region $|x| \leq c_{1} t$ for $t$ sufficiently large. Aronson (1977) proved that an analogous result holds for Kendall's epidemic model.

A steady state of system (3) is given by $S=\sigma, I=R=0$, where $\sigma>0$ is a constant. To study the asymptotic behavior of solutions to system (3), 
consider the initial values

$$
S(x, 0)=\sigma, \quad I(x, 0)=I_{0}(x), \quad R(x, 0)=0, \quad x \in \mathbb{R},
$$

where $I_{0}(x) \geq 0$ is continuous such that $I(x) \not \equiv 0$ and $I(x) \equiv 0$ in $\left[x_{0}, \infty\right)$ for some $x_{0} \in \mathbb{R}$.

By rescaling, the initial value problem can be re-written as

$$
\begin{aligned}
& \frac{\partial S}{\partial t}=-S(x, t) \int_{-\infty}^{\infty} I(y, t) K(x-y) d y, \\
& \frac{\partial I}{\partial t}=S(x, t) \int_{-\infty}^{\infty} I(y, t) K(x-y) d y-\lambda I(x, t), \\
& \frac{\partial R}{\partial t}=\lambda I(x, t), \\
& S(x, 0)=1, \quad I(x, 0)=I_{0}(x), \quad R(x, 0)=0, \quad x \in \mathbb{R},
\end{aligned}
$$

where $\lambda=\gamma / \beta \sigma$. It is not difficult to see that if $(S, I, R)$ is a solution of system (5), then $R$ satisfies

$$
\begin{aligned}
& \frac{\partial R}{\partial t}=-\lambda R(x, t)+\lambda\left[1-\exp \left(-\frac{1}{\lambda} \int_{-\infty}^{\infty} R(y, t) K(x-y) d y\right)\right]+\lambda I_{0}(x), \\
& R(x, 0)=0, \quad x \in \mathbb{R} .
\end{aligned}
$$

Conversely, if $R$ is a solution of the problem (6), then $(S, I, R)$ is a solution of system (5) with

$$
\begin{aligned}
& S=\exp \left(-\frac{1}{\lambda} \int_{-\infty}^{\infty} R(y, t) K(x-y) d y\right) \\
& I=-R+\left[1-\exp \left(-\frac{1}{\lambda} \int_{-\infty}^{\infty} R(y, t) K(x-y) d y\right)\right]+I_{0}(x) .
\end{aligned}
$$

Assume that

(K1) $K$ is a nonnegative even function defined in $\mathbb{R}$ with $\int_{-\infty}^{\infty} K(y) d y=1$.

(K2) There exists a $\nu \in(0, \infty]$ such that $\int_{-\infty}^{\infty} e^{\mu y} K(y) d y<\infty$ for all $\mu \in$ $[0, \nu)$.

(K3) Define $A_{\lambda}(\mu)=\frac{1}{\mu}\left[\int_{-\infty}^{\infty} e^{\mu y} K(y) d y-\lambda\right]$. For each $\lambda<1$ there exists a $\mu^{*}=\mu^{*}(\lambda) \in(0, \nu)$ such that $0<c^{*} \equiv A_{\lambda}\left(\mu^{*}\right)=\inf \left\{A_{\lambda}(\mu): 0<\mu<\nu\right\}$, $A_{\lambda}^{\prime}(\mu)<0$ in $\left(0, \mu^{*}\right)$ and $A_{\lambda}^{\prime}(\mu)>0$ in $\left(\mu^{*}, \nu\right)$.

(K4) For each $\bar{\mu} \in(0, \nu)$ there exists an $r=r(\bar{\mu}) \geq 0$ such that $e^{\mu x} K(x)=$ $\min \left\{e^{\mu y} K(y): y \in[0, x]\right\}$ for all $\mu \in[0, \bar{\mu}]$ and $x \geq r(\bar{\mu})$.

Theorem 3.1 (Aronson 1977) Suppose the kernel $K$ satisfies (K1)-(K4). Let $R(x, t)$ be a solution of the problem (6). If $\lambda \geq 1$, then for every $x \in \mathbb{R}$ and $c \geq 0$,

$$
\lim _{t \rightarrow \infty,|x| \geq c t} R(x, t)=0 .
$$


Theorem 3.1 corresponds to the Kermack-McKendrick threshold result. Roughly speaking, it says that an initial infection (given by $I_{0}(x)$ ) does not propagate if $\lambda=\gamma / \beta \sigma \geq 1$, that is, if the initial density of susceptibles $(\sigma)$ is too low or the removal rate $(\gamma)$ is too high.

The next result shows that the situation is quite different for $\lambda \in(0,1)$.

Theorem 3.2 (Aronson 1977) Suppose the kernel $K$ satisfies (K1)-(K4) and $\lambda \in(0,1)$. Let $R(x, t)$ be a solution of the problem (6).

(i) If $c>c^{*}$, then for every $x \in \mathbb{R}$,

$$
\lim _{t \rightarrow \infty,|x| \geq c t} R(x, t)=0 .
$$

(ii) If $0<c<c^{*}$, then for every $x \in \mathbb{R}$,

$$
\lim _{t \rightarrow \infty,|x| \geq c t} R(x, t)=\alpha(\lambda)
$$

where $\alpha(\lambda)$ is the unique solution of $1-\alpha=e^{-\alpha / \lambda}$ in $(0,1)$.

Theorem 3.2 says that if you travel toward $+\infty$ from any point in $\mathbb{R}$, then you will outrun the infection if your speed exceeds the minimal speed $c^{*}$, but the infection will overtake you if your speed is less than $c^{*}$.

\section{Diekmann-Thieme Model}

Suppose that not all individuals are equally susceptible, but certain traits have a marked influence. Let $S(x, t)$ denote the density of susceptibles at time $t$ and location $x$ and $i(x, t, \theta) d \tau$ be the density of infectives who were infected some time between $t-\theta$ and $t-\theta-d \theta$. Then $I(x, t)=\int_{0}^{\infty} i(x, t, \theta) d \theta$ is the density of infectives at time $t$ and location $x$. Let $A(\theta, x, y)$ represent the expected infectivity of an individual who became infected $\theta$ time units ago while having a trait value $y$ towards a susceptible with trait value $x$. Similar to the Kermack-McKendrick model (1), one has (Diekmannn 1978)

$$
\frac{\partial S}{\partial t}=S(x, t) \int_{\Omega} \int_{0}^{\infty} A(\theta, x, y) \frac{\partial S}{\partial t}(y, t-\theta) d \theta d y
$$

where $\Omega$ denotes the set of trait values. Assume

$$
i(x, 0, \theta)=i_{0}(x, \theta), \quad S(x, 0)=S_{0}(x) .
$$

Then equation (7) can be written as

$$
\frac{\partial S}{\partial t}=S(x, t)\left[\int_{0}^{t} \int_{\Omega} A(\theta, x, y) \frac{\partial S}{\partial t}(y, t-\theta) d y d \theta-h(x, t)\right]
$$


where

$$
h(x, t)=\int_{0}^{\infty} \int_{\Omega} i_{0}(x, \theta) A(t+\theta, x, y) d y d \theta .
$$

Now, assuming $S_{0}(x)>0$ for every $x \in \Omega$ and integrating equation (8) with respect to $t$, one obtains the Diekmann-Thieme model (Diekmann 1978, 1979; Thieme 1977a, 1977b, 1979)

$$
u(x, t)=\int_{0}^{t} \int_{\Omega} g(u(y, t-\theta)) k(\theta, x, y) d y d \theta+f(x, t),
$$

where

$$
\begin{array}{cc}
u(x, t)=-\ln \frac{S(x, t)}{S_{0}(x)}, & g(u)=1-e^{-u}, \\
k(\theta, x, y)=S_{0}(y) A(\theta, x, y), & f(x, t)=\int_{0}^{t} h(x, s) d s .
\end{array}
$$

Let $B C(\Omega)$ be the Banach space of bounded continuous functions on $\Omega$ equipped with the supremum norm. Denote $C_{T}=C([0, T] ; B C(\Omega))$ the Banach space of continuous functions on $[0, T]$ with values in $B C(\Omega)$ equipped with the norm

$$
\|f\|_{C_{T}}=\sup _{0 \leq t \leq T}\|f[t]\|_{B C(\Omega)},
$$

where $f(x, t)$ is written as $f[t]$ when it is regarded as an element of $C_{T}$. The first result is about the local and global existence and uniqueness of the solution of equation (9).

Theorem 4.1 (Diekmann 1978) Suppose $g$ is locally Lipschitz continuous and $f: \mathbb{R}_{+} \rightarrow B C(\Omega)$ is continuous, then there exists a $T>0$ such that equation (9) has a unique solution $u$ in $C_{T}$. If $g$ is uniformly Lipschitz continuous, then equation (9) has a unique solution $u: \mathbb{R}_{+} \rightarrow B C(\Omega)$.

Remark 2. Thieme (1977a) proved a very similar result for a more general model and considered how far an epidemic can spread. See also Thieme (1977b).

The next result is about the positivity, monotonicity and stabilization of the solution of equation (9).

Theorem 4.2 (Diekmann 1978)

(1) Suppose $g(u)>0$ for $u>0$ and $f[t] \geq 0$ for all $t \geq 0$, Then $u[t] \geq 0$ on the domain of definition of $u$.

(2) Suppose, in addition, $g$ is monotone nondecreasing and $f[t+h] \geq f[t]$ for all $h \geq 0$, then $u[t+h] \geq u[t]$ for all $h \geq 0$ and $t \geq 0$ such that $t+h$ is in the domain of definition of $u$. 
(3) Suppose, in addition, that $g$ is bounded and uniformly Lipschitz continuous on $\mathbb{R}_{+}$and that the subset $\{f[t] \mid t \geq 0\}$ of $B C(\Omega)$ is uniformly bounded and equicontinuous and that $k$ satisfies

(i) For each $x \in \Omega, \int_{0}^{t} k(\theta, x, \cdot) d \theta \rightarrow \int_{0}^{\infty} k(\theta, x, \cdot) d \theta$ in $L_{1}(\Omega)$ as $t \rightarrow \infty$, and for some $C>0, \sup _{x \in \Omega} \int_{\Omega} \int_{0}^{\infty} k(\theta, x, y) d \theta d y<C$.

(ii) For each $\varepsilon>0$ there exists $\delta=\delta(\varepsilon)>0$ such that if $x_{1}, x_{2} \in \Omega$ and $\left|x_{1}-x_{2}\right|<\delta$, then $\int_{\Omega} \int_{0}^{\infty}\left|k\left(\theta, x_{1}, y\right)-k\left(\theta, x_{2}, y\right)\right| d \theta d y<\varepsilon$.

Then the solution $u$ of equation (9) is defined on $\mathbb{R}_{+}$and there exists $u[\infty] \in B C(\Omega)$ such that, as $t \rightarrow \infty, u[t] \rightarrow u[\infty]$ in $B C(\Omega)$ if $\Omega$ is compact, and uniformly on compact subset of $\Omega$ if $\Omega$ is not compact. Moreover, $u[\infty]$ satisfies the limit equation

$$
u[\infty]=\int_{\Omega} g(u[\infty](y)) \int_{0}^{\infty} k(\theta, x, y) d \theta d y+f[\infty](x) .
$$

Now consider the Diekmann-Thieme model (9) with $\Omega=\mathbb{R}^{n}(n=1,2,3)$. Assume $k(\theta, x, y)=k(\theta, x-y): \mathbb{R}_{+} \times \mathbb{R}^{n} \rightarrow \mathbb{R}_{+}$is a Borel measurable function satisfying

$(\mathrm{k} 1) k^{*}=\int_{0}^{\infty} \int_{\mathbb{R}^{n}} k(\theta, y) d y d \theta \in(1, \infty)$.

(k2) There exists some $\lambda_{0}>0$ such that $\int_{0}^{\infty} \int_{\mathbb{R}^{n}} e^{\lambda_{0} y_{1}} k(\theta, y) d y d \theta<\infty$, where $y_{1}$ is the first coordinate of $y \in \mathbb{R}^{n}$.

(k3) There are constants $\sigma_{2}>\sigma_{1}>0, \rho>0$ such that $k(\theta, x)>0$ for all $\theta \in\left(\sigma_{1}, \sigma_{2}\right)$ and $|x| \in(0, \rho)$.

(k4) $k$ is isotropic (i.e., $k(\theta, x)=k(\theta, y)$ if $|x|=|y|)$.

Define

$$
c^{*}=\inf \left\{c \geq 0: \int_{0}^{\infty} \int_{\mathbb{R}^{n}} e^{-\lambda\left(c \theta+y_{1}\right)} k(\theta, y) d y d \theta<1 \text { for some } \lambda>0\right\} \text {. }
$$

Assume that $g: \mathbb{R}_{+} \rightarrow \mathbb{R}_{+}$is a Lipschitz continuous function satisfying

(g1) $g(0)=0$ and $g(u)>0$ for all $u>0$.

(g2) $g$ is differentiable at $u=0, g^{\prime}(0)=1$ and $g(u) \leq u$ for all $u>0$.

(g3) $\lim _{u \rightarrow \infty} g(u) / u=0$.

(g4) There exists a positive solution $u^{*}$ of $u=k^{*} g(u)$ such that $k^{*} g(u)>u$ for all $u \in\left(0, u^{*}\right)$ and $k^{*} g(u)<u$ for all $u>u^{*}$.

Thieme (1979) proved that the $c^{*}$ defined by (10) is the asymptotic wave speed (see also Diekmann 1979, Thieme and Zhao 2003).

Theorem 4.3 (Thieme 1979) Assume $k$ satisfies (k1)-(k4) and $g$ satisfies (g1)-(g4).

(i) For every admissible $f(x, t)$, the unique solution $u(x, t)$ of $(9)$ satisfies

$$
\lim _{t \rightarrow \infty,|x| \geq c t} u(x, t)=0
$$

for each $c>c^{*}$. 
(ii) If $g$ is monotone increasing and $f(x, t): \mathbb{R}^{n} \times \mathbb{R}_{+} \rightarrow \mathbb{R}_{+}$is a Borel measurable function such that $f(x, t) \geq \eta>0$ for all $t \in\left(t_{1}, t_{2}\right)$ and $|x| \leq \eta$ with $t_{2}>t_{1} \geq 0, \eta>0$, then

$$
\lim _{t \rightarrow \infty,|x| \geq c t} u(x, t) \geq u^{*}
$$

for each $c \in\left(0, c^{*}\right)$.

To discuss the existence of traveling wave solutions in equation (9), we assume $\Omega=\mathbb{R}$ and $f(x, t)=0$. Suppose $g$ satisfies the modified assumptions:

(g5) $g(0)=0$ and there exists a positive solution $u^{*}$ of $u=k^{*} g(u)$ such that $k^{*} g(u)>u$ for all $u \in\left(0, u^{*}\right)$.

(g6) $g$ is differentiable at $u=0, g^{\prime}(0)=1$ and $g(u) \leq u$ for all $u \in\left[0, u^{*}\right]$.

Theorem 4.4 (Diekmann 1978, 1979) Suppose $k(\theta, x)$ satisfies (k1)-(k4) with $n=1$ and $g$ satisfies (g5)-(g6). Moreover, assume that $g$ is monotone increasing on $\left[0, u^{*}\right]$ and $g(u) \geq u-a u^{2}$ for all $u \in\left[0, u^{*}\right]$ and some $a>0$. Then for each $c>c^{*}$, there exists a monotone traveling wave solution of equation (9) with speed $c$ which connects 0 and $u^{*}$.

Remark 3. Thieme and Zhao (2003) considered a more general nonlinear integral equation and studied the asymptotic speeds of spread and traveling waves. Schumacher (1980a, 1980b) argued that the following model

$$
\frac{\partial u}{\partial t}=\int_{0}^{\infty} \int_{-\infty}^{\infty} g(u(x-y, t-s)) d \eta(y, s)
$$

is more reasonable, where $\eta$ is a Lebesgue measure on $\mathbb{R} \times \mathbb{R}_{+}$such that $\eta(\mathbb{R} \times$ $\left.\mathbb{R}_{+}\right)=1$, and investigated the asymptotic speed of propagation, existence of traveling fronts and dependence of the minimal speed on delays.

\section{Migration and Spatial Spread}

Spatial heterogeneities can be included by adding an immigration term where infective individuals enter the system at a constant rate. This clearly allows the persistence of the disease because if it dies out in one region then the arrival of an infective from elsewhere can trigger another epidemic. Indeed, the arrival of new infectives has been demonstrated as being important in the outbreaks of measles observed in Iceland, a small island community (Cliff et al., 1993). A constant immigration term has a mildly stabilizing effect on the dynamics and tends to increase the minimum number of infective individuals observed in the models (Bolker and Grenfell 1995). De Mottoni et al. (1979) and Busenberg and Travis (1983) considered a population in an open bounded region $\Omega \subset \mathbb{R}^{n}(n \leq 3)$ with smooth boundary $\partial \Omega$ and assumed that the susceptible, infective, and removed individuals can migrate inside the region $\Omega$ according to the rules of group migration. 


\subsection{An SI Model}

Assume the population consists of only two classes, the susceptibles $S(x, t)$ and the infectives $I(x, t)$, at time $t$ and location $x \in \Omega$. Assume that both the susceptibles and infectives can migrate according to a Fickian diffusion law with each subpopulation undergoing a flux which is proportional to the gradient of that particular subpopulation: $\Delta S$ and $d \Delta I$, respectively, where the diffusion rate of the susceptibles is normalized to be one and $d>0$ is the diffusion rate for the infectives. The mechanism of infection is governed by a nonlocal law, as in the Kendall model. It is also assumed that the susceptibles grow at a rate $\mu>0$ the susceptibles are removed (e.g. by vaccination) depending on an effectiveness coefficient $\sigma$. Based on these assumptions, De Mottoni et al. (1979) considered the following model

$$
\begin{aligned}
& \frac{\partial S}{\partial t}=\Delta S+\mu-\sigma S(x, t)-S(x, t) \int_{\Omega} I(y, t) K(x, y) d y \\
& \frac{\partial I}{\partial t}=d \Delta I+S(x, t) \int_{\Omega} I(y, t) K(x, y) d y-\gamma I(x, t)
\end{aligned}
$$

under the boundary value conditions

$$
\frac{\partial S}{\partial \mathbf{n}}(x, t)=\frac{\partial I}{\partial \mathbf{n}}(x, t)=0, \quad(x, t) \in \Omega \times(0, \infty)
$$

and initial value conditions

$$
S(x, 0)=S_{0}(x), \quad I(x, 0)=I_{0}(x), \quad x \in \Omega .
$$

Let $C(\bar{\Omega})$ denote the Banach space of continuous functions on $\bar{\Omega}$ endowed with supremum norm $\|u\|=\max _{x \in \bar{\Omega}}|u(x)|$. Let $X=C(\bar{\Omega}) \oplus C(\bar{\Omega})$ with norm $|U|_{X}=\|u\|+\|v\|$ for $U=(u, v) \in X$.

De Mottoni et al. (1979) proved the following local stability and global attractivity of the disease free equilibrium $(\mu / \sigma, 0)$, where stability is meant relative to the $X$-norm. Thus, the threshold type result has been generalized to the diffusive nonlocal epidemic model (12).

Theorem 5.1 (De Mottoni et al. 1979) Assume that $\mu<\gamma / \sigma$. Then

(i) The steady state solution $(\mu / \sigma, 0)$ is asymptotically stable.

(ii) For any $\left(S_{0}, I_{0}\right) \in X$ with $S_{0} \geq 0, I_{0} \geq 0$, the corresponding solution of (12) converges to $(\mu / \sigma, 0)$ in $X$ as $t \rightarrow \infty$.

When $\mu=\sigma=0, K(\cdot)$ equals $\beta$ times a delta function, system (12) reduces to a reaction-diffusion model of the form

$$
\begin{aligned}
& \frac{\partial S}{\partial t}=\frac{\partial^{2} S}{\partial x^{2}}-\beta S I, \\
& \frac{\partial I}{\partial t}=d \frac{\partial^{2} I}{\partial x^{2}}+\beta S I-\gamma I .
\end{aligned}
$$


Capasso (1979) and Webb (1981) studied the stability of the disease free steady state of the system (15).

To discuss the existence of traveling wave solutions, consider $x \in \mathbb{R}$. Look for traveling wave solutions of the form

$$
S(x, t)=g(\xi), \quad I(x, t)=f(\xi), \quad \xi=x-c t
$$

satisfying

$$
g(-\infty)=\varepsilon\left(\varepsilon<S_{0}\right), \quad g(+\infty)=S_{0}, \quad f(-\infty)=f(+\infty)=0 .
$$

where $c$ is the wave speed to be determined, $\varepsilon$ is some positive constant. The following result was obtained by Hosono and Ilyas (1995).

Theorem 5.2 (Hosono and Ilyas 1995) Assume that $\gamma / \beta S_{0}<1$. Then for each $c \geq c^{*}=2 \sqrt{\beta S_{0} d\left(1-\gamma / \beta S_{0}\right)}$ there exists a positive constant $\varepsilon^{*}$ such that system (15) has a traveling wave solution $(S(x, t), I(x, t))=(g(\xi), f(\xi))$ for $\varepsilon=\varepsilon^{*}$.

Notice that when $\gamma / \beta S_{0}>1$, the system has no traveling wave solutions. The threshold condition $\gamma / \beta S_{0}<1$ for the existence of traveling wave solutions has some implications. We can see that for any epidemic wave to occur, there is a minimum critical density of the susceptible population $S_{c}=\gamma / \beta$. Also, for a given population size $S_{0}$ and mortality rate $\gamma$, there is a critical transmission rate $\beta_{c}=\gamma / S_{0}$. When $\beta>\beta_{c}$, the infection will spread. With a given transmission rate and susceptible population we can also obtain a critical mortality rate $\gamma_{c}=\beta S_{0}$, there is an epidemic wave moving through the population if $\gamma<\gamma_{c}$.

\subsection{An SIR Model}

Assume that a portion of those who are infected acquire immunity to further infection and join the removed class, while the remainder of those who are infected return to the susceptible class and are subject to possible further infections. Busenberg and Travis (1983) derived the following Kendall type SIS model

$$
\begin{aligned}
& \frac{\partial S}{\partial t}=d \frac{S(x, t)}{N(x, t)} \Delta S-S(x, t) \int_{\Omega} I(y, t) K(x, y) d y+\gamma_{1} I(x, t), \\
& \frac{\partial I}{\partial t}=d \frac{I(x, t)}{N(x, t)} \Delta I+S(x, t) \int_{\Omega} I(y, t) K(x, y) d y-\gamma I(x, t), \\
& \frac{\partial R}{\partial t}=d \frac{R(x, t)}{N(x, t)} \Delta I+\gamma_{2} I(x, t)
\end{aligned}
$$

under the boundary value conditions

$$
\frac{\partial S}{\partial \mathbf{n}}(x, t)=\frac{\partial I}{\partial \mathbf{n}}(x, t)=\frac{\partial R}{\partial \mathbf{n}}(x, t)=0, \quad(x, t) \in \partial \Omega \times(0, \infty)
$$


and initial value conditions

$$
S(x, 0)=S_{0}(x), \quad I(x, 0)=I_{0}(x), \quad R(x, 0)=R_{0}(x), \quad x \in \Omega .
$$

$N(x, t)=S(x, t)+I(x, t)+R(x, t)$ satisfies the linear initial-boundary value problem

$$
\begin{aligned}
& \frac{\partial N}{\partial t}=d \Delta N(x, t), \\
& \frac{\partial N}{\partial \mathbf{n}}(x, t)=0, \quad(x, t) \in \partial \Omega \times(0, \infty), \\
& N(x, 0)=S_{0}(x)+I_{0}(x)+R_{0}(x), \quad x \in \Omega .
\end{aligned}
$$

Theorem 5.3 (Busenberg and Travis 1983) Let $K(x, y)>0$ be twice continuously differentiable on $\bar{\Omega} \times \bar{\Omega}$, and let $S_{0}>0, I_{0}>0, R_{0}>0$ be twice continuously differentiable with the sum $N_{0}$ satisfying the Neumann condition in (20). Then the problem (17) - (19) has a unique positive solution $(S(x, t), I(x, t), R(x, t))$ for $(x, t) \in \Omega \times \mathbb{R}_{+}$. Moreover,

$$
\lim _{t \rightarrow \infty}(S(x, t), I(x, t), R(x, t))=(\hat{S}(x), \hat{I}(x), \hat{R}(x)),
$$

where

$$
\begin{aligned}
& \hat{S}(x)=\frac{a_{0}\left(N_{0}(x)-R_{0}(x)\right)}{N_{0}(x)}-a_{0} \gamma_{2} \int_{0}^{\infty} \frac{I(x, s)}{N(x, s)} d s, \\
& \hat{I}(x)=0 \\
& \hat{R}(x)=\frac{a_{0} R_{0}(x)}{N_{0}(x)}+a_{0} \gamma_{2} \int_{0}^{\infty} \frac{I(x, s)}{N(x, s)} d s
\end{aligned}
$$

and $a_{0}=\int_{\Omega} N_{0}(x) d x / \int_{\Omega} d x$.

The result indicates that a portion of those who are infected eventually acquire immunity, and the only possible limit is one where the disease dies out. The steady state distribution of the susceptible and immune subpopulations is generally spatially non-uniform and depends on the initial distributions of the different subclasses. It also depends on the time history of the evolution of the proportion $I(x, t) / N(x, t)$ of infected individuals through the integral $a_{0} \gamma_{2} \int_{0}^{\infty} I(x, s) / N(x, s) d s$, which represents that portion of the infected subpopulation at position $x \in \Omega$ which becomes immune during the span of the epidemic.

\section{A Vector-Disease Model}

We consider a host-vector model for a disease without immunity in which the current density of infectious vectors is related to the number of infectious hosts at earlier times. Spatial spread in a region is modeled by a diffusion term. Consider a host in a bounded region $\Omega \subset \mathbb{R}^{n}(n \leq 3)$ where a disease 
(malaria) is carried by a vector (mosquito). The host is divided into two classes, susceptible and infectious, whereas the vector population is divided into three classes, infectious, exposed, and susceptible. Suppose that the infection in the host confers negligible immunity and does not result death or isolation. All new-borns are susceptible. The host population is assumed to be stable, that is, the birth rate is constant and equal to the death rate. Moreover, the total host population is homogeneously distributed in $\Omega$ and both susceptible and infectious populations are allowed to diffuse inside $\Omega$, however, there is no migration through $\partial \Omega$, the boundary of $\Omega$.

For the transmission of the disease, it is assumed that a susceptible host can receive the infection only by contacting with infected vectors, and a susceptible vector can receive the infection only from the infectious host. Also, a susceptible vector becomes exposed when it receives the infection from an infected host. It remains exposed for some time and then becomes infectious. The total vector population is also constant and homogeneous in $\Omega$. All three vector classes diffuse inside $\Omega$ and cannot cross the boundary of $\Omega$.

Denote by $u(t, x)$ and $v(t, x)$ the normalized spatial density of infectious and susceptible host at time $t$ in $x$, respectively, where the normalization is done with respect to the spatial density of the total population. Hence, we have

$$
u(t, x)+v(t, x)=1, \quad(t, x) \in \mathbb{R}_{+} \times \Omega .
$$

Similarly, define $I(t, x)$ and $S(t, x)$ as the normalized spatial density of infectious and susceptible vector at time $t$ in $x$, respectively.

If $\alpha$ denotes the host-vector contact rate, then the density of new infections in host is given by

$$
\alpha v(t, x) I(t, x)=\alpha[1-u(t, x)] I(t, x) .
$$

The density of infections vanishes at a rate $a u(t, x)$, where $a$ is the cure/recovery rate of the infected host. The difference of host densities of arriving and leaving infections per unit time is given by $d \Delta u(t, x)$, where $d$ is the diffusion constant, $\Delta$ is the Laplacian operator. We then obtain the following equation

$$
\frac{\partial u}{\partial t}(t, x)=d \Delta u(t, x)-a u(t, x)+\alpha[1-u(t, x)] I(t, x) .
$$

If the vector population is large enough, we can assume that the density of vectors which become exposed at time $t$ in $x \in \Omega$ is proportional to the density of the infectious hosts at time $t$ in $x$. That is, $S(t, x)=h u(t, x)$, where $h$ is a positive constant. Let $\xi(t, s, x, y)$ denote the proportion of vectors which arrive in $x$ at time $t$, starting from $y$ at time $t-s$, then

$$
\int_{\Omega} \xi(t, s, x, y) S(t-s, y) d y
$$


is the density of vectors which became exposed at time $t-s$ and are in $x$ at time $t$. Let $\eta(s)$ be the proportion of vectors which are still infectious $s$ units of time after they became exposed, then

$$
\begin{aligned}
I(t, x) & =\int_{0}^{\infty} \int_{\Omega} \xi(t, s, x, y) S(t-s, y) \eta(s) d y d s \\
& =\int_{0}^{\infty} \int_{\Omega} \xi(t, s, x, y) h \eta(s) u(t-s, y) d y d s .
\end{aligned}
$$

Substituting $I(t, x)$ into equation (21), changing the limits, and denoting

$$
b=\alpha h, \quad F(t, s, x, y)=\xi(t, s, x, y) \eta(s),
$$

we obtain the following diffusive integro-differential equation modeling the vector disease

$$
\frac{\partial u}{\partial t}(t, x)=d \Delta u(t, x)-a u(t, x)+b[1-u(t, x)] \int_{-\infty}^{t} \int_{\Omega} F(t, s, x, y) u(s, y) d y d s
$$

for $(t, x) \in I \mathbb{R}_{+} \times \Omega$. The initial value condition is given by

$$
u(\theta, x)=\phi(\theta, x), \quad(\theta, x) \in(-\infty, 0] \times \Omega,
$$

where $\phi$ is a continuous function for $(\theta, x) \in(-\infty, 0] \times \Omega$, and the boundary value condition is given by

$$
\frac{\partial u}{\partial n}(t, x)=0, \quad(t, x) \in \mathbb{R}_{+} \times \partial \Omega,
$$

where $\partial / \partial n$ represents the outward normal derivative on $\partial \Omega$.

The convolution kernel $F(t, s, x, y)$ is a positive continuous function in its variables $t \in \mathbb{R}, s \in \mathbb{R}_{+}, x, y \in \Omega$. We normalize the kernel so that

$$
\int_{0}^{\infty} \int_{\Omega} F(t, s, x, y) d y d s=1 .
$$

Various types of equations can be derived from equation (22) by taking different kernels.

(i) If $F(t, s, x, y)=\delta(x-y) G(t, s)$, then equation (22) becomes the following integro-differential equation with a local delay

$$
\frac{\partial u}{\partial t}=d \Delta u(t, x)-a u(t, x)+b[1-u(t, x)] \int_{-\infty}^{t} G(t-s) u(s, x) d s
$$

for $(t, x) \in \mathbb{R}_{+} \times \Omega$.

(ii) If $F(t, s, x, y)=\delta(x-y) \delta(t-s)$, then equation (22) becomes the following reaction diffusion equation without delay 


$$
\frac{\partial u}{\partial t}=d \Delta u(t, x)-a u(t, x)+b[1-u(t, x)] u(t, x), \quad(t, x) \in \mathbb{R}_{+} \times \Omega .
$$

(iii) If $F(t, s, x, y)=\delta(x-y) \delta(t-s-\tau)$, where $\tau>0$ is a constant, and $u$ does not depend on the spatial variable, then equation (22) becomes the following ordinary differential equation with a constant delay

$$
\frac{d u}{d t}=-a u(t)+b[1-u(t)] u(t-\tau) .
$$

Cooke (1977) studied the stability of equation (27) and showed that when $0<b \leq a$, then the trivial equilibrium $u_{0}=0$ is globally stable; when $0 \leq a<b$, the trivial equilibrium is unstable and the positive equilibrium $u_{1}=(b-a) / b$ is globally stable. Busenberg and Cooke (1978) assumed that the coefficients are periodic and investigated the existence and stability of periodic solutions of the equation (27). Thieme (1988) considered equation (27) when the coefficients are time-dependent and showed that, under suitable assumptions, the following dichotomy holds: either all non-negative solutions converge to zero or all pairs of non-negative solutions $u(t)$ and $v(t)$ with non-zero initial data satisfy $u(t) / v(t) \rightarrow 0$ as $t \rightarrow \infty$. The case with multiple groups and distributed risk of infection was studied by Thieme (1985). Marcati and Pozio (1983) proved the global stability of the constant solution to (22) when the delay is finite. Volz (1982) assumed that all coefficients are periodic and discussed the existence and stability of periodic solutions of equation (22).

We first consider the stability of steady states of equation (22) with general kernel. Then we discuss the existence of traveling wave solutions in the equation when the kernel takes some specific forms.

\subsection{Stability of the Steady States}

Denote $E=C(\bar{\Omega}, \mathbb{R})$. Then $E$ is a Banach space with respect to the norm

$$
|u|_{E}=\max _{x \in \bar{\Omega}}|u(x)|, \quad u \in E .
$$

Denote $\mathcal{C}=B C((-\infty, 0], E)$. For $\phi \in \mathcal{C}$, define

$$
\|\phi\|=\sup _{\theta \in(-\infty, 0]}|\phi(\theta)|_{E} .
$$

For any $\beta \in(0, \infty)$, if $u:(-\infty, \beta) \rightarrow E$ is a continuous function, $u_{t}$ is defined by $u_{t}(\theta)=u(t+\theta), \quad \theta \in(-\infty, 0]$.

Define

$$
\begin{aligned}
& \mathcal{D}(A)=\left\{u \in E: \quad \Delta u \in E, \quad \frac{\partial u}{\partial n}=0 \text { on } \partial \Omega\right\} \\
& A u=d \Delta u \text { for all } u \in \mathcal{D}(A) \\
& f(\phi)(x)=-a \phi(0, x)+b[1-\phi(0, x)] \int_{-\infty}^{0} \int_{\Omega} F(0, s, x, y) \phi(s, y) d y d s
\end{aligned}
$$


where $\phi \in \mathcal{C}, x \in \bar{\Omega}$. Then we can re-write equation (22) into the following abstract form:

$$
\begin{aligned}
& \frac{d u}{d t}=A u+f\left(u_{t}\right), \quad t \geq 0, \\
& u_{0}=\phi \in \mathcal{C},
\end{aligned}
$$

where

(a) $A: \mathcal{D}(A) \rightarrow E$ is the infinitesimal generator of a strongly continuous semigroup $e^{t A}$ for $t \geq 0$ on $E$ endowed with the maximum norm;

(b) $f: \mathcal{C} \rightarrow E$ is Lipschitz continuous on bounded sets of $\mathcal{C}$.

Associated to equation (28), we also consider the following integral equation

$$
\begin{aligned}
& u(t)=e^{t A} \phi(0)+\int_{0}^{t} e^{(t-s) A} f\left(u_{s}\right) d s, \quad t \geq 0, \\
& u_{0}=\phi
\end{aligned}
$$

A continuous solution of the integral equation (29) is called a mild solution to the abstract equation (28). The existence and uniqueness of the maximal mild solution to equation (28) follow from a standard argument (see Ruan and $\mathrm{Wu}(1994)$ and $\mathrm{Wu}(1996)$ ). When the initial value is taken inside an invariant bounded set in $\mathcal{C}$, the boundedness of the maximal mild solution implies the global existence.

Define

$$
M=\{u \in E: 0 \leq u(x) \leq 1, x \in \bar{\Omega}\} .
$$

We can prove that $M$ is invariant by using the results on invariance and attractivity of sets for general partial functional differential equations established by Pozio $(1980,1983)$ and follow the arguments in Marcati and Pozio (1980).

Theorem 6.1 (Ruan and Xiao 2004) The set $M$ is invariant; that is, if $\phi \in B C((-\infty, 0] ; M)$ then $u(\phi)$ exists globally and $u(\phi)(t) \in M$ for all $t \geq 0$.

The stability of the steady state solutions can be established following the attractivity results of Pozio $(1980,1983)$.

Theorem 6.2 (Ruan and Xiao 2004) The following statements hold

(i) If $0<b \leq a$, then $u_{0}=0$ is the unique steady state solution of (22) in $M$ and it is globally asymptotically stable in $B C((-\infty, 0] ; M)$.

(ii) If $0 \leq a<b$, then there are two steady state solutions in $M$ : $u_{0}=0$ and $u_{1}=(b-a) / b$, where $u_{0}$ is unstable and $u_{1}$ is globally asymptotically stable in $B C((-\infty, 0] ; M)$.

Recall that $b$ represents the contact rate and $a$ represents the recovery rate. The stability results indicate that there is a threshold at $b=a$. If $b \leq a$, then the proportion $u$ of infectious individuals tends to zero as $t$ becomes large and the disease dies out. If $b>a$, the proportion of infectious 
individuals tends to an endemic level $u_{1}=(b-a) / b$ as $t$ becomes large. There is no non-constant periodic solutions in the region $0 \leq u \leq 1$.

The above results also apply to the special cases (25), (26), and (27) and thus include the following results on global stability of the steady states of the discrete delay model (27) obtained by Cooke (1977) (using the Liapunov functional method).

Corollary 6.3 (Cooke 1977) For the discrete delay model (27), we have the following statements

(i) If $0<b \leq a$, then the steady state solution $u_{0}=0$ is asymptotically stable and the set $\{\phi \in C([-\tau, 0], \mathbb{R}): 0 \leq \phi(\theta) \leq 1$ for $-\tau \leq \theta \leq 0\}$ is a region of attraction.

(ii) If $0 \leq a<b$, then the steady state solution $u_{1}=(b-a) / b$ is asymptotically stable and the set $\{\phi \in C([-\tau, 0], \mathbb{R}): 0<\phi(\theta) \leq 1$ for $-\tau \leq \theta \leq 0\}$ is a region of attraction.

\subsection{Existence of Traveling Waves}

We know that when $b>a$ equation (22) has two steady state solutions, $u_{0}=0$ and $u_{1}=(b-a) / b$. In this section we consider $x \in(-\infty, \infty)$ and establish the existence of traveling wave solutions of the form $u(x, t)=U(z)$ such that

$$
\lim _{z \rightarrow-\infty} U(z)=\frac{b-a}{b}, \quad \lim _{z \rightarrow \infty} U(z)=0,
$$

where $z=x-c t$ is the wave variable, $c \geq 0$ is the wave speed. Consider two cases: (a) without delay, i.e, equation (26); (b) with local delay, i.e., equation (25). We scale the model so that $d=1$.

(a) Without Delay. Substitute $u(x, t)=U(z)$ into the reaction diffusion equation (26) without delay, i.e.,

$$
\frac{\partial u}{\partial t}=\Delta u(t, x)-a u(t, x)+b[1-u(t, x)] u(t, x),
$$

we obtain the traveling wave equation

$$
U^{\prime \prime}+c U^{\prime}+(b-a-b U) U=0,
$$

which is equivalent to the following system of first order equations

$$
\begin{aligned}
& U^{\prime}=V, \\
& V^{\prime}=-c V-(b-a-b U) U .
\end{aligned}
$$

System (30) has two equilibria: $E_{0}=(0,0)$ and $E_{1}=((b-a) / b, 0)$. The following result shows that there is a traveling front solution of equation (30) connecting $E_{0}$ and $E_{1}$. 
Theorem 6.4 If $c \geq 2 \sqrt{b-a}$, then in the $(U, V)$ phase plane for system (30) there is a heteroclinic orbit connecting the critical points $E_{0}$ and $E_{1}$. The heteroclinic connection is confined to $V<0$ and the traveling wave $U(z)$ is strictly monotonically decreasing.

(b) With Local Delay. Consider the diffusive integro-differential equation (25) with a local delay kernel

$$
G(t)=\frac{t}{\tau^{2}} e^{-t / \tau}
$$

which is called the strong kernel. The parameter $\tau>0$ measures the delay, which implies that a particular time in the past, namely $\tau$ time units ago, is more important than any other since the kernel achieves its unique maximum when $t=\tau$. Equation (25) becomes

$$
\frac{\partial u}{\partial t}=\Delta u(t, x)-a u(t, x)+b[1-u(t, x)] \int_{-\infty}^{t} \frac{t-s}{\tau^{2}} e^{-\frac{t-s}{\tau}} u(s, x) d s
$$

for $(t, x) \in \mathbb{R}_{+} \times \Omega$. Define $U(z)=u(x, t)$ and

$$
W(z)=\int_{0}^{\infty} \frac{t}{\tau^{2}} e^{-t / \tau} U(z+c t) d t, \quad Y(z)=\int_{0}^{\infty} \frac{1}{\tau} e^{-t / \tau} U(z+c t) d t .
$$

Differentiating with respect to $z$ and denoting $U^{\prime}=V$, we obtain the following traveling wave equations

$$
\begin{aligned}
& U^{\prime}=V, \\
& V^{\prime}=a U-c V-b W+b U W, \\
& c \tau W^{\prime}=W-Y, \\
& c \tau Y^{\prime}=-U+Y .
\end{aligned}
$$

For $\tau>0$, system (32) has two equilibria

$$
(0,0,0,0) \quad \text { and } \quad\left(\frac{b-a}{b}, 0, \frac{b-a}{b}, \frac{b-a}{b}\right) .
$$

A traveling front solution of the original equation exists if there exists a heteroclinic orbit connecting these two critical points.

Note that when $\tau$ is very small, system (32) is a singularly perturbed system. Let $z=\tau \eta$. Then system (32) becomes

$$
\begin{aligned}
& \dot{U}=\tau V, \\
& \dot{V}=\tau(a U-c V-b W+b U W), \\
& c \dot{W}=W-Y, \\
& c \dot{Y}=-U+Y,
\end{aligned}
$$

where dots denote differentiation with respect to $\eta$. While these two systems are equivalent for $\tau>0$, the different time scales give rise to two different limiting systems. Letting $\tau \rightarrow 0$ in (32), we obtain 


$$
\begin{aligned}
& \dot{U}=\tau V, \\
& \dot{V}=\tau(a U-c V-b W+b U W), \\
& 0=W-Y, \\
& 0=-U+Y .
\end{aligned}
$$

Thus, the flow of system (34) is confined to the set

$$
\mathcal{M}_{0}=\left\{(U, V, W, Y) \in \mathbb{R}^{4}: W=U, Y=U\right\},
$$

and its dynamics are determined by the first two equations only. On the other hand, setting $\tau \rightarrow 0$ in (33) results in the system

$$
\begin{aligned}
& U^{\prime}=0 \\
& V^{\prime}=0 \\
& c W^{\prime}=W-Y, \\
& c Y^{\prime}=-U+Y
\end{aligned}
$$

Any points in $\mathcal{M}_{0}$ are the equilibria of system (36). Generally, (32) is referred to as the slow system since the time scale $z$ is slow, and (33) is referred to as the fast system since the time scale $\eta$ is fast. Hence, $U$ and $V$ are called slow variables and $W$ and $Y$ are called the fast variables. $\mathcal{M}_{0}$ is the slow manifold.

If $\mathcal{M}_{0}$ is normally hyperbolic, then we can use the geometric singular perturbation theory of Fenichel (1979) to obtain a two-dimensional invariant manifold $\mathcal{M}_{\tau}$ for the flow when $0<\tau \ll 1$, which implies the persistence of the slow manifold as well as the stable and unstable foliations. As a consequence, the dynamics in the vicinity of the slow manifold are completely determined by the one on the slow manifold. Therefore, we only need to study the flow of the slow system (32) restricted to $\mathcal{M}_{\tau}$ and show that the two-dimensional reduced system has a heteroclinic orbit.

Recall that $\mathcal{M}_{0}$ is a normally hyperbolic manifold if the linearization of the fast system (33), restricted to $\mathcal{M}_{0}$, has exactly $\operatorname{dim} \mathcal{M}_{0}$ eigenvalues with zero real part. The eigenvalues of the linearization of the fast system restricted to $\mathcal{M}_{0}$ are $0,0,1 / c, 1 / c$. Thus, $\mathcal{M}_{0}$ is normally hyperbolic.

The geometric singular perturbation theorem now implies that there exists a two-dimensional manifold $\mathcal{M}_{\tau}$ for $\tau>0$. To determine $\mathcal{M}_{\tau}$ explicitly, we have

$$
\mathcal{M}_{\tau}=\left\{(U, V, W, Y) \in \mathbb{R}^{4}: W=U+g(U, V ; \tau), \quad Y=U+h(U, V ; \tau)\right\},
$$

where the functions $g$ and $h$ are to be determined and satisfy

$$
g(U, V ; 0)=h(U, V ; 0)=0 .
$$

By substituting into the slow system (32), we know that $g$ and $h$ satisfy 


$$
\begin{aligned}
& c \tau\left[\left(1+\frac{\partial h}{\partial U}+\frac{\partial g}{\partial U}\right) V+\left(\frac{\partial h}{\partial V}+\frac{\partial g}{\partial V}\right)(a U-\right. c V-b(U+h+g) \\
&+b U(U+h+g))]=g, \\
& c \tau\left[\left(1+\frac{\partial h}{\partial U}\right) V+\frac{\partial h}{\partial V}(a U-c V-b(U+h+g)\right. \\
&+b U(U+h+g))]=h .
\end{aligned}
$$

Since $h$ and $g$ are zero when $\tau=0$, we set

$$
\begin{aligned}
& g(U, V ; \tau)=\tau g_{1}(U, V)+\tau^{2} g_{2}(U, V)+\cdots, \\
& h(U, V ; \tau)=\tau h_{1}(U, V)+\tau^{2} h_{2}(U, V)+\cdots .
\end{aligned}
$$

Substituting $g(U, V ; \tau)$ and $h(U, V ; \tau)$ into the above equations and comparing powers of $\tau$, we obtain

$$
\begin{aligned}
& g_{1}(U, V)=c V \\
& h_{1}(U, V)=c V \\
& g_{2}(U, V)=2 c^{2}(a U-c V-b(1-U) U), \\
& h_{2}(U, V)=c^{2}(a U-c V-b(1-U) U) .
\end{aligned}
$$

The slow system (32) restricted to $\mathcal{M}_{\tau}$ is therefore given by

$$
\begin{aligned}
& U^{\prime}=V \\
& V^{\prime}=a U-c V-b(1-U)[U+g(U, V ; \tau)+h(U, V ; \tau)],
\end{aligned}
$$

where $g$ and $h$ are given by (39) and (40). Note that when $\tau=0$ system (41) reduces to the corresponding system (30) for the nondelay equation. We can see that for $0<\tau \ll 1$ system (41) still has critical points $E_{0}$ and $E_{1}$. The following theorem shows that there is a heteroclinic orbit connecting $E_{0}$ and $E_{1}$ and thus equation (31) has a traveling wave solution connecting $u_{0}=0$ and $u_{1}=(b-a) / b$.

Theorem 6.5 (Ruan and Xiao 2004) For any $\tau>0$ sufficiently small there exist a speed $c$ such that the system (41) has a heteroclinic orbit connecting the two equilibrium points $E_{0}$ and $E_{1}$.

The above results (Theorems 6.4 and 6.5) show that for the small delay the traveling waves are qualitatively similar to those of the non-delay equation. The existence of traveling front solutions show that there is a moving zone of transition from the disease-free state to the infective state.

Remark 4. When the delay kernel is non-local, for example,

$$
F(x, t)=\frac{1}{\tau_{0}} e^{-\frac{t}{\tau_{0}}} \frac{1}{\sqrt{4 \pi \rho_{0}}} e^{-\frac{x^{2}}{4 \rho_{0}}}, \tau_{0}>0, \rho_{0}>,
$$

the existence of traveling wave solutions in equation (22) can be established by using the results in Wang, Li and Ruan (2005). 


\section{Discussion}

Epidemic theory for homogeneous populations has shown that a critical quantity, known as the basic reproductive value (which maybe considered as the fitness of a pathogen in a given population), must be greater than unity for the pathogen to invade a susceptible population (Anderson and May 1991). In reality, populations tend not to be homogeneous and there are nonlocal interactions. Therefore, there has been much theoretical investigation on the geographical spread of infectious diseases.

Invasion of diseases is now an international public health problem. The mechanisms of invasion of diseases to new territories may take many different forms and there are several ways to model such problems. One way is to introduce spatial effects into the model, divide the population into $n$ subpopulations and allow infective individuals in one patch to infect susceptible individuals in another. The equilibrium behavior of such models has been studied widely, see Lajmanovich and Yorke (1976), Hethcote (1978), Dushoff and Levin (1995), Lloyd and May (1996), etc. It has been shown that spatial heterogeneity can reduce the occurrence of fade-outs in epidemic models (Bolker and Grenfell 1995).

Another way is to assume that there are nonlocal interactions between the susceptible and infective individuals and use integral equations to model the epidemics. In this short survey, we focused on the spatiotemporal dynamics of some nonlocal epidemiological models, include the classical KermackMcKendrick model, the Kendall model given by differential and integral equations, the Diekmann-Thieme model described by a double integral equation, the diffusive integral equations proposed by De Mottoni et al. (1979) and Busenberg and Travis (1983), a vector-disease model described by a diffusive double integral equation (Ruan and Xiao (2004)), etc.

For some diseases, such as vector-host diseases, the infectives at location $x$ at the present time $t$ were infected at another location $y$ at an earlier time $t-s$. In order to study the effect of spatial heterogeneity (geographical movement), nonlocal interactions and time delay (latent period) on the spread of the disease, it is reasonable to consider more general models of the following form

$$
\begin{aligned}
& \frac{\partial S}{\partial t}=d_{1} \Delta S-S(x, t) \int_{-\infty}^{t} \int_{\Omega} I(y, s) K(x, y, t-s) d y d s \\
& \frac{\partial I}{\partial t}=d_{2} \Delta I+S(x, t) \int_{-\infty}^{t} \int_{\Omega} I(y, s) K(x, y, t-s) d y d s-\gamma I(x, t) \\
& \frac{\partial R}{\partial t}=d_{3} \Delta I+\gamma I(x, t)
\end{aligned}
$$

under certain boundary and initial conditions, where $d_{1}, d_{2}, d_{2}$ are the diffusion rates for the susceptible, infective, and removed individuals, respectively. The kernel $K(x, y, t-s) \geq 0$ describes the interaction between the infective 
and susceptible individuals at location $x \in \Omega$ at the present time $t$ which occurred at location $y \in \Omega$ at an earlier time $t-s$. It will be very interesting to study the spatiotemporal dynamics, such as stability of the disease-free equilibrium and existence of traveling waves, in the general model (42) and apply the results to study the geographical spread of some vector-borne diseases, such as West Nile virus and malaria.

Acknowledgments. I am grateful to Horst Thieme for giving me some very helpful comments and remarks on an earlier version of the paper and for bringing several references to my attention. I also would like to thank Odo Diekmann, Nick Britton and the referee for making some valuable comments and suggestions.

\section{References}

1. R. M. Anderson and R. M. May (1991), Infectious Diseases of Humans: Dynamics and Control, Oxford University Press, Oxford.

2. D. G. Aronson (1977), The asymptotic speed of propagation of a simple epidemic, in "Nonlinear Diffusion", eds. by W. E. Fitzgibbon and H. F. Walker, Research Notes in Math. 14, Pitman, London, pp. 1-23.

3. D. G. Aronson and H. F. Weinberger (1975), Nonlinear diffusion in population genetics, combustion, and nerve pulse propagation, in "Partial Differential Equations and Related Topics", ed. by J. A. Goldstein, Lecture Notes in Math. 446, Springer, Berlin, pp. 5-49.

4. D. G. Aronson and H. F. Weinberger (1975), Multidimensional nonlinear diffusion arising in population genetics, Adv. Math. 30: 33-76.

5. C. Atkinson and G. E. H. Reuter (1976), Deterministic epidemic waves, Math. Proc. Camb. Phil. Soc. 80: 315-330.

6. A. D. Barbour (1977), The uniqueness of Atkinson and Reuter's epidemic waves, Math. Proc. Camb. Phil. Soc. 82: 127-130.

7. M. S. Bartlett (1956), Deterministic and stochastic models for recurrent epidemics, Proc. 3rd Berkeley Symp. Math. Stat. Prob. 4: 81-109.

8. B. M. Bolker and B. T. Grenfell (1995), Space, persistence, and dynamics of measles epidemics, Phil. Trans. R. Soc. Lond. B237: 298-219.

9. F. Brauer and C. Castillo-Chavez (2000), Mathematical Models in Population Biology and Epidemiology, Springer-Verlag, New York.

10. K. J. Brown and J. Carr (1977), Deterministic epidemic waves of critical velocity, Math. Proc. Camb. Phil. Soc. 81: 431-433.

11. S. Busenberg and K. L. Cooke (1978), Periodic solutions for a vector disease model, SIAM J. Appl. Math. 35: 704-721.

12. T. Caraco, S. Glavanakov, G. Chen, J. E. Flaherty, T. K. Ohsumi and B. K. Szymanski (2002), Stage-structured infection transmission and a spatial epidemic: A model for lyme disease, Am. Nat. 160: 348-359.

13. V. Capasso (1978), Global solution for a diffusive nonlinear deterministic epidemic model, SIAM J. Appl. Math. 35: 274-284.

14. A. Cliff, P. Haggett and M. Smallman-Raynor (1993), Measles: An Historical Geography of a Major Human Viral Disease, Blackwell, Oxford. 
15. K. L. Cooke (1979), Stability analysis for a vector disease model, Rocky Mountain J. Math. 9: 31-42.

16. I. Cruickshank, W. S. Gurney and A. R. Veitch (1999), The characteristics of epidemics and invasions with thresholds, Theor. Pop. Biol. 56: 279-92.

17. P. de Monttoni, E. Orlandi and A. Tesei (1979), Asymptotic behavior for a system describing epidemics with migration and spatial spread of infection, Nonlinear Anal. 3: 663-675.

18. O. Diekmann (1978), Thresholds and travelling waves for the geographical spread of infection, J. Math. Biol. 6: 109-130.

19. O. Diekmann (1979), Run for life: A note on the asymptotic spread of propagation of an epidemic, J. Differential Equations 33: 58-73.

20. O. Diekmann and J. A. P. Heesterbeek (2000), Mathematical Epidemiology of Infective Diseases: Model Building, Analysis and Interpretation, Wiley, New York.

21. O. Diekmann, J. A. P. Heesterbeek and H. Metz (1995), The legacy of Kermack and McKendrick, in "Epidemic Models: Their Structure and Relation to Data", ed. by D. Mollison, Cambridge Univ. Press, Cambridge, pp. 95-115.

22. J. Dushoff and S. A. Levin (1995), The effects of population heterogeneity on disease invasion, Math. Biosci. 128: 25-40.

23. N. Fenichel (1979), Geometric singular perturbation theory for ordinary differential equations. J. Differential Equations 31: 53-98.

24. R. A. Fisher (1937), The wave of advance of advantageous genes, Ann. Eugenics 7: $353-369$.

25. B. T. Grenfell, O. N. Bjornstad and J. Kappey (2001), Travelling waves and spatial hierarchies in measles epidemics, Nature 414: 716-723.

26. H. W. Hethcote (2000), The mathematics of infectious disease, SIAM Rev. 42: 599-653.

27. Y. Hosono and B. Ilyas (1995), Traveling waves for a simple diffusive epidemic model, Math. Models Methods Appl. Sci. 5: 935-966.

28. A. Källen, P. Arcuri and J. D. Murray (1985), A simple model for the spatial spread and control of rabies, J. Theor. Biol. 116: 377-393.

29. D. G. Kendall (1957), Discussion of 'Measles periodicity and community size' by M. S. Bartlett, J. Roy. Stat. Soc. A120: 64-76.

30. W. O. Kermack and A. G. McKendrik (1927), A contribution to the mathematical theory of epidemics, Proc. Roy. Soc. A115: 700-721.

31. A. Kolmogorov, I. Petrovskii, and N. S. Piskunov (1937), Étude de l'équation de le diffusion avec croissance de la quantité de mateère et son application à un problème biologique, Moscow Univ. Bull. 1: 1-25.

32. A. Lajmanovich and J. A. Yorke (1976), A deterministic model for gonorrhea in a non-homogeneous population, Math. Biosci. 28: 221-236.

33. A. L. Lloyd and R. M. May (1996), Spatial heterogeneity in epidemic models, J. Theor. Biol. 179: 1-11.

34. L. P. Lounibos (2002), Invasions by insect vectors of human disease, Ann. Rev. Entomol. 47: 233-266.

35. P. Marcati and M. A. Pozio (1980), Global asymptotic stability for a vector disease model with spatial spread, J. Math. Biol. 9: 179-187.

36. J. Medlock and M. Kot (2003), Spreading disease: integro-differential equations old and new, Math. Biosci. 184: 201-222.

37. D. Mollison (1972), Possible velocities for a simple epidemic, Adv. Appl. Prob. 4: 233-257. 
38. D. Mollison(1991), Dependence of epidemic and population velocities on basic parameters, Math. Biosci. 107: 255-87.

39. J. D. Murray (1989), Mathematical Biology, Springer-Verlag, Berlin.

40. J. D. Murray and W. L. Seward (1992), On the spatial spread of rabies among foxes with immunity, J. Theor. Biol. 156: 327-348.

41. J. D. Murray, E. A. Stanley and D. L. Brown (1986), On the spatial spread of rabies among foxes, Proc. R. Soc. Lond. B229(1255): 111-150.

42. M. O'Callagham and A. G. Murray (2002), A tractable deterministic model with realistic latent periodic for an epidemic in a linear habitat, J. Math. Biol. 44: 227-251.

43. M. A. Pozio (1980), Behavior of solutions of some abstract functional differential equations and application to predator-prey dynamics, Nonlinear Anal. 4: 917-938.

44. M. A. Pozio (1983), Some conditions for global asymptotic stability of equilibria of integrodifferential equations, J. Math. Anal. Appl. 95: 501-527.

45. L. Rass and J. Radcliffe (2003), Spatial Deterministic Epidemics, Math. Surveys Monogr. 102, Amer. Math. Soc., Providence.

46. R. Ross and H. P. Hudson (1917), An application of the theory of probability to the study of a priori pathometry - Part III, Proc. R. Soc. Lond. A93: 225-240.

47. S. Ruan and J. Wu (1994), Reaction-diffusion equations with infinite delay, Can. Appl. Math. Quart. 2: 485-550.

48. S. Ruan and D. Xiao (2004), Stability of steady states and existence of traveling waves in a vector disease model, Proc. Royal Soc. Edinburgh Sect. A134: 9911011.

49. K. Schumacher (1980a), Travelling-front solution for integro-differential equation. I, J. Reine Angew. Math. 316: 54-70.

50. K. Schumacher (1980b), Travelling-front solution for integro-differential equation. II, in "Biological Growth and Spread", Lecture Notes in Biomathematics 38, eds. by W. Jäger, H. Rost and P. Tautu, Springer, Berlin, pp. 296-309.

51. H. R. Thieme (1977a), A model for the spatial spread of an epidemic, J. Math. Biol. 4: 337-351.

52. H. R. Thieme (1977b), The asymptotic behavior of solutions of nonlinear integral equations, Math. Zeitchrift 157: 141-154.

53. H. R. Thieme (1979), Asymptotic estimates of the solutions of nonlinear integral equation and asymptotic speeds for the spread of populations, J. Reine Angew. Math. 306: 94-121.

54. H. R. Thieme (1980), Some mathematical considerations of how to stop the spatial spread of a rabies epidemics, in "Biological Growth and Spread," Lecture Notes in Biomathematics 38, eds. by W. Jäger, H. Rost and P. Tautu, Springer, Berlin, pp. 310-319.

55. H. R. Thieme (1985), Renewal theorems for some mathematical models in epidemiology, J. Integral Equations 8: 185-216.

56. H. R. Thieme (1988), Asymptotic proportionality (weak ergodicity) and conditional asymptotic equality of solutions to time-heterogeneous sublinear difference and differential equations, J. Differential Equations 73: 237-268.

57. H. R. Thieme (2003), Mathematics in Population Biology, Princeton University Press, Princeton.

58. H. R. Thieme and X.-Q. Zhao (2003), Asymptotic speeds of spread and traveling waves for integral equations and delayed reaction-diffusion models, J. Differential Equations 195: 430-470. 
59. F. van den Bosch, J. A. J. Metz and O. Diekmann (1990), The velocity of spatial population expansion, J. Math. Biol. 28: 529-565.

60. R. Volz (1982), Global asymptotic stability of a periodic solution to an epidemic model, J. Math. Biol. 15: 319-338.

61. Z.-C. Wang, W.-T. Li and S. Ruan (2005), Traveling wave fronts in reactiondiffusion systems with spatio-temporal delays, J. Differential Equations (in press).

62. G. F. Webb (1981), A reaction-diffusion model for a deterministic diffusive epidemic, J. Math. Anal. Appl. 84 (1981), 150-161.

63. J. Wu (1996), Theory and Applications of Partial Functional Differential Equations, Springer, New York. 